\title{
Synthesis and optical studies of gamma irradiated Eu doped nanocrystalline $\mathrm{CaF}_{2}$
}

\author{
C. Pandurangappa ${ }^{\mathrm{a}, *}$, B.N. Lakshminarasappa $^{\mathrm{b}}$, B.M. Nagabhushana ${ }^{\mathrm{c}}$ \\ a Department of Physics, RNS Institute of Technology, Channasandra, Bangalore 560061, India \\ ${ }^{\mathrm{b}}$ Department of Physics, Jnanabharathi Campus, Bangalore University, Bangalore 560056, India \\ c Department of Chemistry, M.S. Ramaiah Institute of Technology, Bangalore 560054, India
}

\section{A R T I C L E I N F O}

\section{Article history:}

Received 30 January 2011

Received in revised form 20 March 2011

Accepted 28 April 2011

Available online 7 May 2011

\section{Keywords:}

Nanostructured materials

Crystal structure

Point defects

Scanning electron microscopy

Luminescence

X-ray diffraction

\begin{abstract}
A B S T R A C T
Europium (Eu) doped Calcium fluoride $\left(\mathrm{CaF}_{2}\right)$ nanoparticles are synthesized by co-precipitation method and characterized by powder X-ray diffraction (PXRD), Fourier transform infrared (FTIR) spectroscopy and scanning electron microscopy (SEM). Also, optical absorption and photoluminescence (PL) results on gamma irradiated Eu doped $\mathrm{CaF}_{2}$ nanoparticles are presented. The PXRD patterns confirmed the cubic crystallinity of the samples and the particle size is found to be $\sim 25 \mathrm{~nm}$. The purity of the synthesized nanoparticles is confirmed from FTIR spectrum. The morphological features studied using SEM revealed that nanoparticles are agglomerated and porous. Optical absorption spectrum shows that $\gamma$-rayed Eu doped $\mathrm{CaF}_{2}$ nanoparticles exhibit absorption bands at $\sim 279,360$ and $434 \mathrm{~nm}$. The PL studies showed emission at $\sim 420,525$ and $552 \mathrm{~nm}$.
\end{abstract}

(C) 2011 Elsevier B.V. All rights reserved.

\section{Introduction}

Nanomaterials compose a unique class of materials that have reduced dimensions, typically within the range of 1-100 nm. Nanomaterials are strongly influenced by the surface characteristics which differ from their bulk counterparts. The loss of the threedimensional periodicity of atoms in nanophase materials results in structural modifications. These changes are known to alter electronic properties and chemical reactivity. Also, surface modification of insulating nanoparticles can lead to enhanced luminescence efficiency. Fluoride nanomaterials form the subject of interest because of their potential technological applications. In spite of their attractive structural and optical characteristics nanofluorides have not received as much attention as other optical and optoelectronic nanomaterials like semiconductors. Synthesis of luminescent materials in nanophase can change the properties and efficiency of the materials for optical applications [1]. There are number of well known and newly developed methods for preparation of nanoparticles [2]. One of the fluorides with a wide transparent spectral range $(200-1100 \mathrm{~nm})$ and large band gap $(\sim 12 \mathrm{eV})$ is Calcium fluoride $\left(\mathrm{CaF}_{2}\right) \cdot \mathrm{CaF}_{2}$ gained a renewed interest as a laser material when doped with rare earth materials [3]. $\mathrm{CaF}_{2}$

\footnotetext{
* Corresponding author. Tel.: +91 97418 54545; fax: +91 8028611882.

E-mail addresses: cpandu@gmail.com, cpandurangappa@yahoo.com (C. Pandurangappa).
}

nanoparticles are synthesized by different methods such as solgel, solvothermal, hydrothermal, reverse micelle [4-6]. In the present work Eu (1-5 mol\%) doped $\mathrm{CaF}_{2}$ nanoparticles are synthesized by co-precipitation method and characterized by XRD, FTIR and SEM. Also, optical absorption and Photoluminescence studies on gamma irradiated ( $\gamma$-rayed) Eu doped $(2 \mathrm{~mol} \%)$ nanoparticles are carried out.

\section{Experimental}

Stoichiometric quantity of europium oxide $\left(\mathrm{Eu}_{2} \mathrm{O}_{3}\right)$ was taken in a $250 \mathrm{ml}$ conical flask and small quantity of nitric acid $\left(1: 1 \mathrm{HNO}_{3}\right)$ was added in to it. The mixture was evaporated slowly on a sand bath to convert the $\mathrm{Eu}_{2} \mathrm{O}_{3}$ into europium nitrate $\left(\mathrm{Eu}\left(\mathrm{NO}_{3}\right)_{2}\right)$. Calcium chloride $\left(\mathrm{CaCl}_{2}\right)$ and ammonium fluoride $\left(\mathrm{NH}_{4} \mathrm{~F}\right)$ were added into the conical flask in stoichiometric quantities and the mixture was dissolved in $100 \mathrm{ml}$ distilled water. The mixed solution was stirred constantly for $2 \mathrm{~h}$ using a magnetic stirrer to achieve homogeneity. While stirring the transparent reaction mixture transformed into opaque white suspension gradually. The solution was centrifuged for $10 \mathrm{~min}$ at $5000 \mathrm{rpm}$ and a white residue was obtained. The residue was washed thoroughly with ethanol to remove the residual chloride and the ammonium ions. The product was extracted on to a ceramic dish and dried slowly on a sand bath maintained at $\sim 100^{\circ} \mathrm{C}$.

The XRD measurements of synthesized samples were carried out using Philips $\mathrm{X}$-pert PRO powder diffractometer with $\mathrm{Cu}-\mathrm{K}_{\alpha}$ radiation $(\lambda=1.54056 \AA)$ in the scan range $10-90^{\circ}$ at a low speed of $\sim 1^{\circ} / \mathrm{min}$. The morphology of synthesized samples was studied using scanning electron microscope (JEOL JSM-840A) by sputtering technique with gold as covering contrast material. The FTIR spectrum was recorded using Nicolet Magna 550 spectrometer with $\mathrm{KBr}$ pellets. The synthesized samples were heat treated at $500{ }^{\circ} \mathrm{C}$ for $2 \mathrm{~h}$. $2 \mathrm{~mol} \%$ Eu doped $\mathrm{CaF}_{2}$ samples were exposed to $\gamma$-rays from a $\mathrm{Co}^{60}$ source at an activity $3.89 \mathrm{KGy} / \mathrm{h}$. The $\gamma$-dose range was 32.4 Gy-15.56 KGy. The Optical absorption measurements of the $\gamma$-rayed samples 


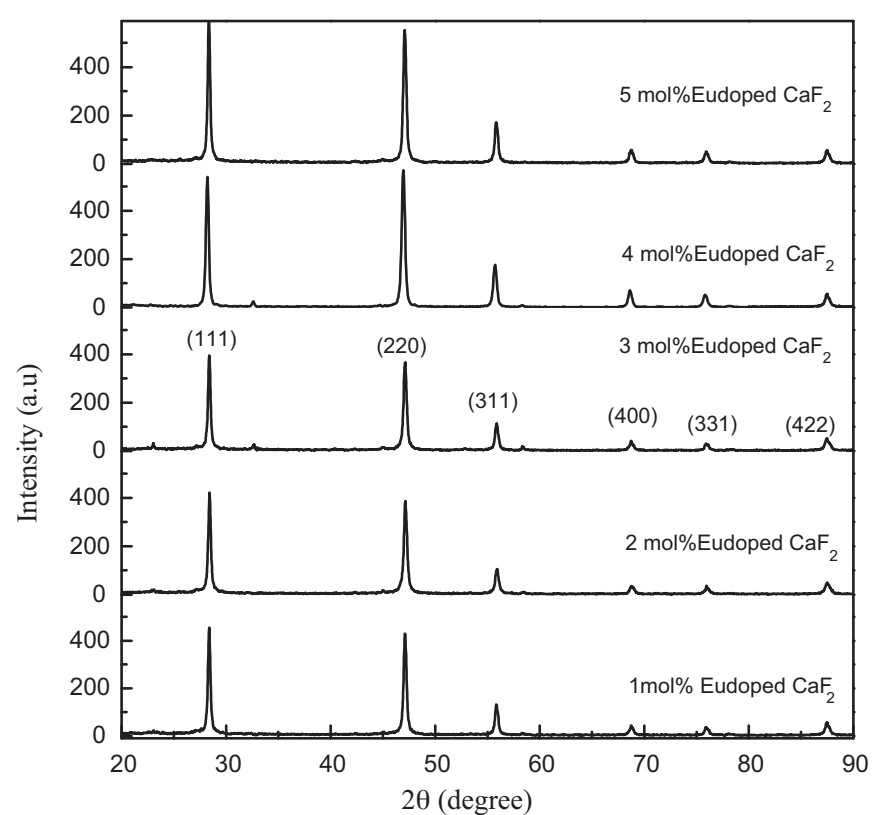

Fig. 1. Powder XRD spectrum of Eu doped nanocrystalline $\mathrm{CaF}_{2}$.

were carried out in the wavelength range 200-900 nm using V-570 UV/VIS/NIR double beam spectrophotometer. The PL emission spectra of the samples were recorded at room temperature using a Fluorolog 3 Jobin Yvon spectrofluoremeter (FL3-11) equipped with a $450 \mathrm{~W}$ Xenon lamp as the excitation source.

\section{Results and discussion}

\subsection{PXRD, SEM and FTIR}

The PXRD pattern of $\mathrm{Eu}\left(1-5 \mathrm{~mol} \%\right.$ ) doped nanocrystalline $\mathrm{CaF}_{2}$ is shown in Fig. 1. The pattern was compared with JCPDS Card no. 87-0971. All the XRD peaks obtained were indexed and it resulted in a cubic phase of the fluorite structure with space group $\mathrm{Fm} 3 \mathrm{~m}$ [7]. The average value of lattice constant was found to be $5.478 \AA$. The crystallite size calculated using Scherer's formula was $\sim 25 \mathrm{~nm}$. From the figure it is clear that the increase in concentration of Eu from $1-5 \mathrm{~mol} \%$ did not cause any structural changes. The PXRD pattern of $500{ }^{\circ} \mathrm{C}$ heat treated samples was found to be similar to that of as prepared one with a marginal increase in intensity. This indicates that $500{ }^{\circ} \mathrm{C}$ heat treatment does not reveal any structural changes in the nanocrystalline $\mathrm{CaF}_{2}$. However, the crystallite size

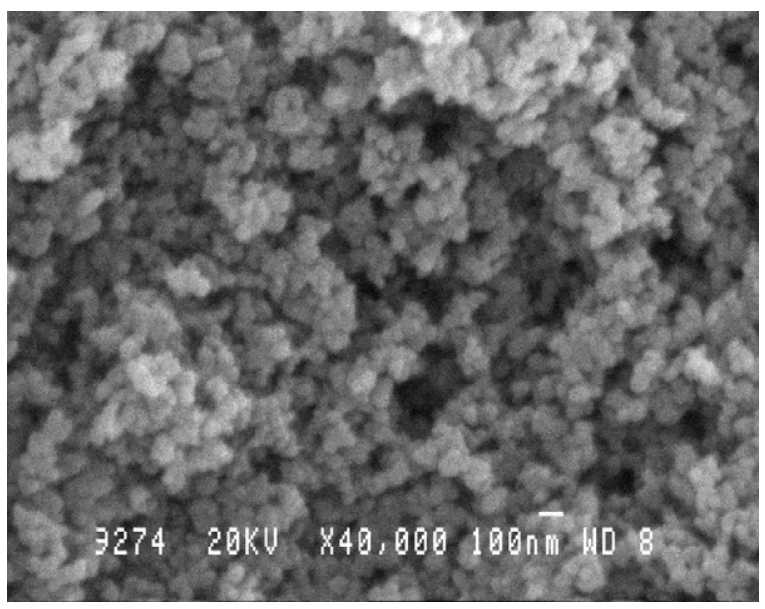

Fig. 2. SEM picture of Eu doped ( $2 \mathrm{~mol} \%$ ) nanocrystalline $\mathrm{CaF}_{2}$.

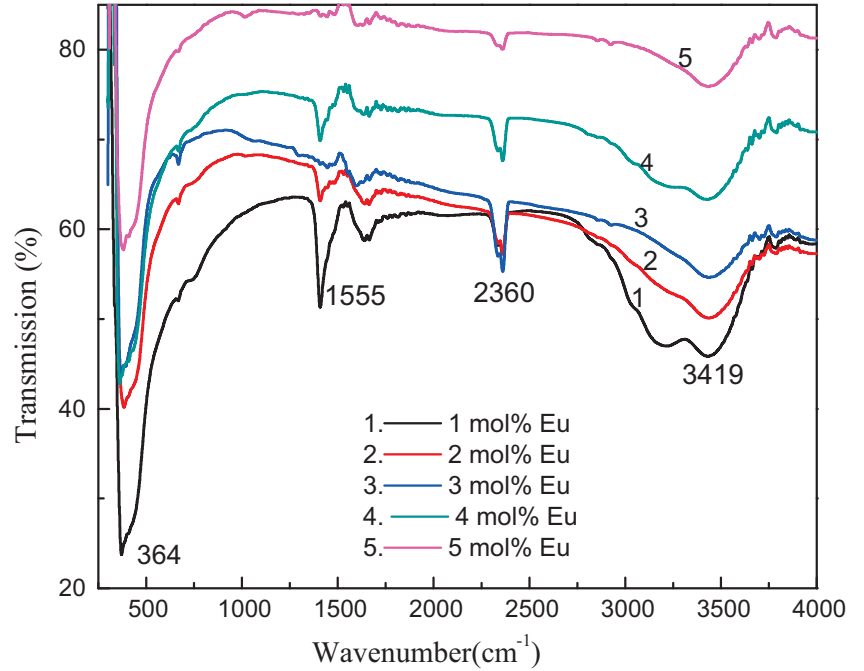

Fig. 3. FTIR spectra of Eu doped nanocrystalline $\mathrm{CaF}_{2}$.

of heat treated samples was found to be increased to $30 \mathrm{~nm}$. This indicates the agglomeration of nanoparticles due to heat treatment.

Fig. 2 shows the SEM photograph of as prepared Eu doped ( $2 \mathrm{~mol} \%$ ) nanocrystalline $\mathrm{CaF}_{2}$. The SEM pictures indicate that the as prepared nanoparticles are agglomerated from few microns to few tens of microns. They are fluffy and porous in nature. The presence of voids in the as prepared nanoparticles can be clearly seen from SEM picture. Fig. 3 shows the FTIR spectra of Eu doped (1-5 mol\%) nanocrystalline $\mathrm{CaF}_{2}$. The absorptions bands at 3400 and $1550 \mathrm{~cm}^{-1}$ are characteristic of $\mathrm{H}-\mathrm{O}-\mathrm{H}$ bending of the $\mathrm{H}_{2} \mathrm{O}$ molecules [9]. It is clear from the figure that the five curves corresponding to different concentrations are similar. This confirms the iso-structural nature of the $1-5 \mathrm{~mol} \%$ Eu doped $\mathrm{CaF}_{2}$ nanoparticles [8]. With increase in $\mathrm{mol} \%$ of Eu there is no considerable change in the position of bands. However, the intensity of the bands vary with increase in Eu concentration. It is well established that in the FTIR spectrum of a solid the band width depends on the nature of chemical bond, bond strength and presence of defects in the sample. A small change in the bond strength shifts the position of absorption bands and changes their intensity. In Eu doped $\mathrm{CaF}_{2}$ crystal the doped $\mathrm{Eu}^{3+}$ ions substitute some of the $\mathrm{Ca}^{2+}$ ions in the $\mathrm{CaF}_{2}$ lattice. This results

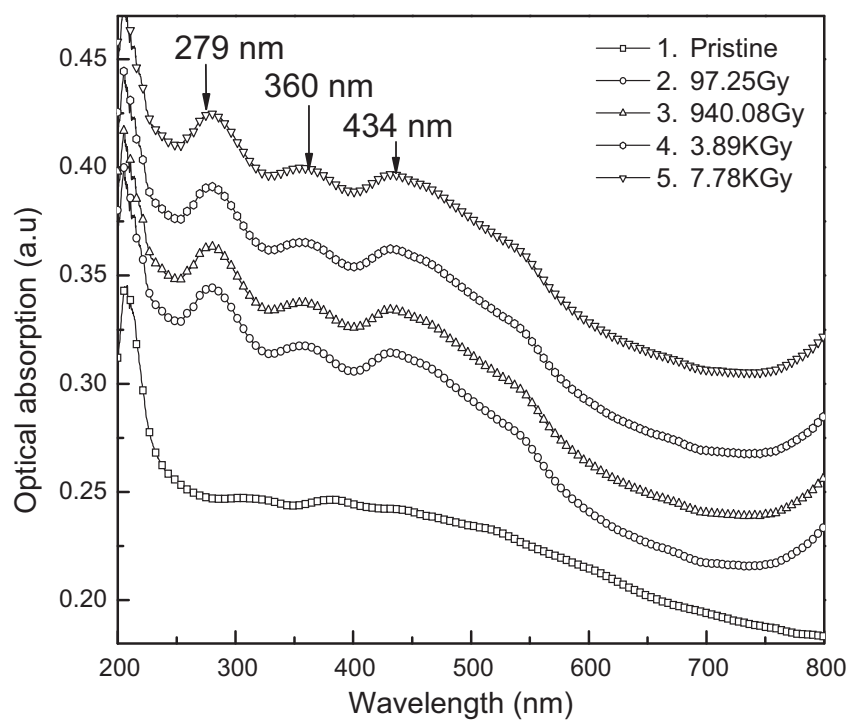

Fig. 4. Optical absorption spectrum of $\gamma$-rayed Eu doped nanocrystalline $\mathrm{CaF}_{2}$. 


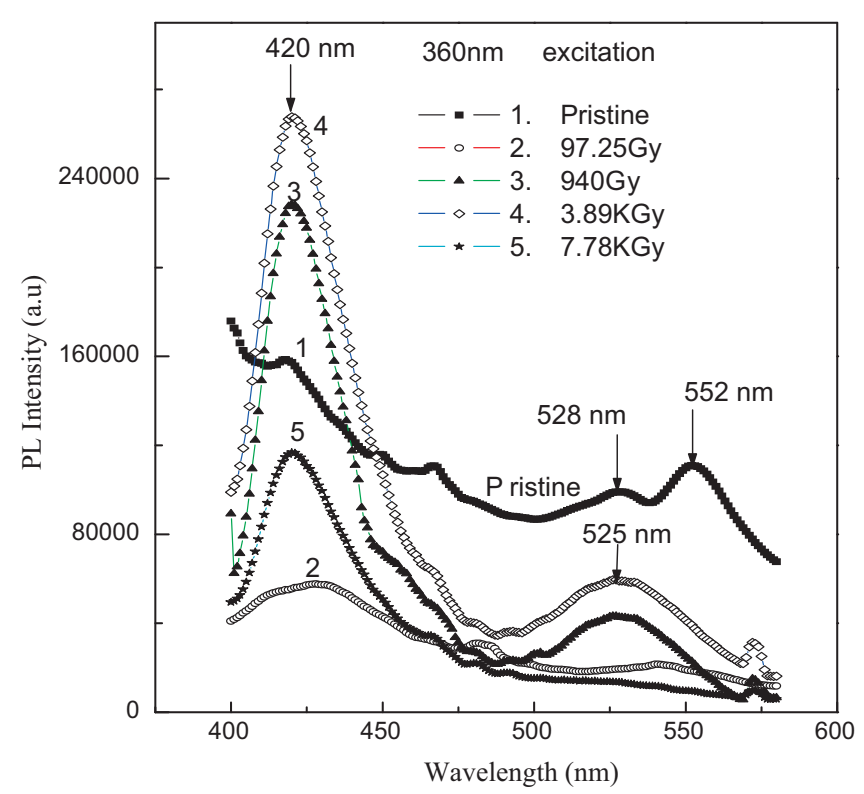

Fig. 5. Photoluminescence spectrum of $\gamma$-rayed Eu doped nanocrystalline $\mathrm{CaF}_{2}$.

in the local elastic deformation in the $\mathrm{CaF}_{2}$ lattice. As the concentration of Eu increases from $1 \mathrm{~mol} \%$ to $5 \mathrm{~mol} \%$, more number of $\mathrm{Ca}^{2+}$ ions are replaced by $\mathrm{Eu}^{3+}$ ions. Hence, the frequency of vibration of distorted lattice changes with increase in Eu concentration. It was observed that in case of $500^{\circ} \mathrm{C}$ heat treated samples the intensity of 3400 and $1550 \mathrm{~cm}^{-1}$ bands was decreased. It could be attributed to the release of water molecules trapped inside the solid matrix of the sample.

The variation of back ground intensity with Eu concentration can be accounted in the following way. With increase of Eu concentration from $1 \mathrm{~mol} \%$ to $5 \mathrm{~mol} \%$ the surface hydroxyl groups in the host $\left(\mathrm{CaF}_{2}\right)$ lattice are said to increase [10]. The increase in number of hydroxyl groups increase the intensity of FTIR absorption bands. Also, the strength of the absorption bands depends on the concentration of the dopant [11]. Increase in the dopant concentration causes reduction in the absorption and enhances the transmittance. Thus with increase in Eu concentration the back ground intensity decreases and the transmittance increases.

\subsection{Optical absorption spectra}

The optical absorption spectrum of pristine and $\gamma$-rayed Eu doped ( $2 \mathrm{~mol} \%) \mathrm{CaF}_{2}$ nanoparticles is shown in Fig. 4 . The $500{ }^{\circ} \mathrm{C}$ heat treated pristine sample showed minimal absorption and indicated the defect free state of the nanoparticles. However, $\gamma$-rayed Eu doped $\mathrm{CaF}_{2}$ samples showed three absorption peaks at $\sim 279$, 360 and $434 \mathrm{~nm}$. The optical absorption increased marginally with increase in $\gamma$-dose. But the peak positions are unaltered with increase in dose. The presence of these peaks indicate that defects are created in nanocrystalline $\mathrm{CaF}_{2}$ upon their exposure to $\gamma$ radiations. The absorption peak at $279 \mathrm{~nm}$ in the present study may be attributed to surface defects such as Schottky or Frenkel defects in nanocrystalline $\mathrm{CaF}_{2}$ [12]. The $360 \mathrm{~nm}$ absorption peak is attributed to F-center. It is known that $\gamma$-irradiation produces free electrons which when trapped at negative ion vacancies form F-center [13]. The peak at $434 \mathrm{~nm}$ is assigned to H-center which is a hole connected with neutral fluorine atoms in interstitial positions [14]. The increase in the absorption with $\gamma$-dose indicates the increase in the concentration of defects with dose.

\subsection{Photoluminescence spectra}

The PL emission spectrum of 2 mol\% Eu doped $\mathrm{CaF}_{2}$ nanoparticles is shown in Fig. 5. The pristine samples showed a prominent emission with peak at $\sim 552 \mathrm{~nm}$ and three weak ones at $\sim 420$ and $528 \mathrm{~nm}$ when the samples were excited at $360 \mathrm{~nm}$. However, $\gamma$ rayed samples showed a strong emission with peak at $\sim 420 \mathrm{~nm}$ and a weak one at $\sim 525 \mathrm{~nm}$ for the same excitation $(360 \mathrm{~nm})$. The PL intensity of $420 \mathrm{~nm}$ peak increased with $\gamma$-dose till 3.89 KGy and decreased with further increase in dose. However, in case of $525 \mathrm{~nm}$ peak the PL intensity decreased drastically after 3.89 KGy.

The $\gamma$-irradiation of Eu doped $\mathrm{CaF}_{2}$ samples reduces $\mathrm{Eu}^{3+}$ ions to $\mathrm{Eu}^{2+}$ state. However, at low $\gamma$-dose there will be considerable number of Europium ions existing in the $\mathrm{Eu}^{3+}$ state [15]. The existence of $\mathrm{Eu}^{3+}$ ions is revealed by the characteristic $\mathrm{Eu}^{3+}$ emission peak of at $525 \mathrm{~nm}$ in the irradiated samples. With increase in $\gamma$-dose majority of $\mathrm{Eu}^{3+}$ ions get reduced to $\mathrm{Eu}^{2+}$ state. Thus the number of $\mathrm{Eu}^{3+}$ ions decreased. Hence, the intensity of $525 \mathrm{~nm}$ peak decreased for higher $\gamma$-dose. The emission peak at $420 \mathrm{~nm}$ in the irradiated samples is attributed to $4 \mathrm{f}^{7} \rightarrow 4 \mathrm{f}^{6} 5 \mathrm{~d}^{1}$ transitions of $\mathrm{Eu}^{2+}$ ions [16]. The $552 \mathrm{~nm}$ emission observed in the pristine sample is attributed to the well studied $5 \mathrm{D}_{0} \rightarrow 7 \mathrm{~F}_{0}$ transition of $\mathrm{Eu}^{3+}$ ions [17]. The PL intensity is found to be enhanced in the Eu doped nanocrystals when compared with the undoped $\mathrm{CaF}_{2}$ nanocrystals studied by the present authors.

\section{Conclusions}

Eu doped $\mathrm{CaF}_{2}$ nanoparticles synthesized by co-precipitation method showed particle size of $\sim 25 \mathrm{~nm}$. The morphological features showed that the as prepared samples were agglomerated from few microns to few tens of microns, fluffy and porous. The FTIR spectrum revealed the presence of hydroxyl groups in the as prepared sample. The increase in mol percent of Eu does not cause any structural changes. Optical absorption spectra revealed the presence of number of defect centers in the $\gamma$-irradiated $\mathrm{CaF}_{2}$ nanoparticles. The PL results indicate that $\gamma$-irradiation reduces Eu ions from $\mathrm{Eu}^{3+}$ to $\mathrm{Eu}^{2+}$ state and various intrinsic transitions of $\mathrm{Eu}^{2+}$ ions are possible in the Eu doped nanocrystalline $\mathrm{CaF}_{2}$.

\section{References}

[1] M. Ratner, D. Ratner, Nano Technology, Pearson Pte LTD., 2003.

[2] J. Rodríguez, M.F. García, Synthesis, Properties and Applications of Oxide Nanomaterials, Wiley-Interscience, New Jersey, 2007.

[3] V. Petit, J.L. Doualan, P. Camy, V. Menard, R. Moncorge, Appl. Phys. B 78 (2004) 681-684.

[4] G.A. Kumar, C.W. Chen, J. Ballato, R.E. Riman, Chem. Mater. 19 (2007) 1523-1528.

[5] A. Bensalah, M. Mortier, G. Patriarcheb, P. Gredin, D. Vivien, J. Solid State Chem. 179 (2006) 2636-2644.

[6] C. Cao, W. Qin, J. Zhang, Y. Wang, G. Wang, G. Wei, P. Zhu, L. Wang, L. Jin, Opt. Commun. 281 (2008) 1716-1719.

[7] S. Fujihara, J. Sol-Gel Sci. Technol. 24 (2002) 147-154.

[8] B.-C. Hong, K. Kawano, J. Alloys Compd. 451 (2008) 276-279.

[9] X. Sun, Y. Li, Chem. Commun. 14 (2003) 1768-1769.

[10] M. Hamadanian, A. Reisi-Vanani, A. Majedi, Iran. Chem. Soc. 7 (2010) S52-S58.

[11] R. Ullah, J. Dutta, Proceed Int. Conf. Emer. Technol. (2007) 306-311.

[12] Xiaoming Zhang, Zewei Quan 1, Jun Yang, Piaoping Yang 1, Hongzhou Lian, Jun Lin, Nanotechnology 19 (2008) 075603, 1-8.

[13] D.W. Cooke, B.L. Bennett, J. Nucl. Mater. 321 (2003) 158-164.

[14] X. Xiang, X.T. Zu, S. Zhu, T.H. Ding, L.M. Wang, Opt. Mater. 28 (2006) 930-934.

[15] S.M. Dhopte, P.L. Muthal, V.K. Kondawar, S.V. Moharil, J. Lumin. 54 (1992) 95-101.

[16] J.L. Guerie, P. Gredin, M. Mortier, G. Patriarche, A. de Kozak, Z. Anorg. Allg. Chem. 632 (2006) 1538-1543.

[17] J. Sils, S. Hausfeld, W. Claub, U. Pahl, R. Lindner, M. Reichling, J. Appl. Phys. 106 (2009) 063109, 1-8. 